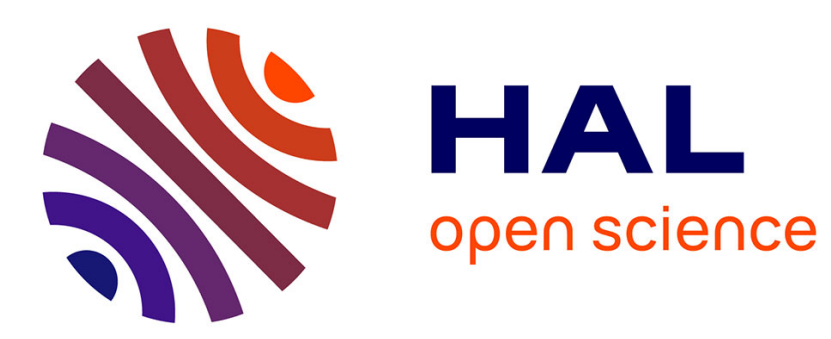

\title{
Etude de la prolificité en épis du maïs dans le premier cycle de sélection de deux populations précoces
}

\author{
Yves Barrière, Patrick Vincourt, Yves Montalant
}

\section{To cite this version:}

Yves Barrière, Patrick Vincourt, Yves Montalant. Etude de la prolificité en épis du maïs dans le premier cycle de sélection de deux populations précoces. Agronomie, 1984, 4 (4), pp.355-360. hal-00884645

\section{HAL Id: hal-00884645 \\ https://hal.science/hal-00884645}

Submitted on 1 Jan 1984

HAL is a multi-disciplinary open access archive for the deposit and dissemination of scientific research documents, whether they are published or not. The documents may come from teaching and research institutions in France or abroad, or from public or private research centers.
L'archive ouverte pluridisciplinaire HAL, est destinée au dépôt et à la diffusion de documents scientifiques de niveau recherche, publiés ou non, émanant des établissements d'enseignement et de recherche français ou étrangers, des laboratoires publics ou privés. 


\title{
Etude de la prolificité en épis du maïs dans le premier cycle de sélection de deux populations précoces
}

\author{
Yves BARRIÈRE $\left({ }^{\mathbf{l}}\right)$, Patrick VINCOURT \& Yves MONTALANT $\left({ }^{*}\right)$ \\ I.N.R.A., Station d'Amélioration des Plantes fourragères, F 86600 Lusignan \\ (*) Station d'Amélioration des Plantes, F 80200 Péronne
}

RÉSUMÉ

\begin{abstract}
Deux populations de maïs précoces prolifiques en épis, à grains respectivement cornés et dentés, ont été constituées en croisement pyramidal entre des lignées précoces non prolifiques et des lignées plus tardives prolifiques, suivi de deux intercroisements. L'étude a porté sur les descendances demi-frères des plantes $\mathrm{S}_{0}$ prolifiques et précoces sélectionnées dans chacune des deux populations, avec une densité de culture de 80000 plantes par hectare. Le niveau de prolificité moyen des familles obtenues est de 1,4 épi par plante, avec une variabilité plus élevée pour la population « cornée ». Les plantes prolifiques se caractérisent par la présence d'une feuille supplémentaire située sous l'épi supérieur, caractère lié à la tardiveté chez les familles « cornées " seulement. L'héritabilité (au sens strict) de la prolificité, calculée pour la population "dentée », n'est pas très élevée $\left(\mathrm{h}^{2}=0,25\right)$. Toutefois, une sélection épi-ligne pratiquée avec un effectif de 20 plantes par familles peut permettre un tri relativement efficace, avec un gain génétique attendu voisin de 0,12 épi/plante par cycle pour un taux de sélection voisin de $30 \mathrm{p}$. 100. La limite actuelle à l'utilisation de ce matériel est liée à sa sensibilité à la verse en végétation, défaut qui devra être corrigé au cours des cycles ultérieurs de sélection.
\end{abstract}

Mots clés additionnels : Héritabilité, index de sélection.

Study of prolificacy in the first selection cycle of two early maize populations.

Two synthetics of early prolific maize, one flint and one dent, were constructed by crossing early nonprolific inbreds and mid-late prolific inbreds (tabl. 1), and intercrossing the complex hybrids obtained twice in isolation. This study deals with the half-sib progenies of early prolific $S_{0}$ plants selected in the second intercrossing of each synthetic. The population density was 80000 plants per ha. The average prolificacy of each synthetic was 1.4 ear per plants with a higher variability of the flint material (tabl. 2). Prolific plants (fig. 1) had one leaf more than non-prolific ones, this leaf being situated below the first ear. The number of leaves above the first ears was about the same in non-prolific and prolific materials, with a tendency for 0.25 leaf less in prolific dent material. PHILIPPE (1980) obtained similar results with later families, and SORRELS et al. (1979), observed a significant positive correlation between prolificacy and number of nodes below the first ear, and a negative one with the number of nodes above the first ear. Prolificacy was negatively correlated with earliness only for the flint synthetic. The narrow-sense heritability of prolificacy, computed for dent material, was not very high, about 0.25 . Nevertheless an ear-to-row selection, with about 20 plants per family, could lead to efficient breeding with a genetic gain of about $0.12 \mathrm{ear} / \mathrm{plant}$ per cycle for a selection rate of $30 \%$. Both synthetics used were susceptible to lodging, which must be corrected during further breeding cycles.

Additional key words : Heritability, selection index.

\section{INTRODUCTION}

L'obtention de variétés prolifiques en épis est une des voies qui devrait permettre d'améliorer la régularité et la productivité des cultures tant de maïs grain que de maïs fourrage (RUSSEL, 1968 ; RUSSEL \& EBE-

( $\left.{ }^{1}\right)$ Ce travail a été réalisé à la Station d'Amélioration des Plantes, I.N.R.A., 80200 Péronne.
RHART, 1968 ; EL-LAKANY \& RUSSEL, 1971 ; HALLAUER \& TROYER, 1972 ; PRIOR \& RUSSEL, 1975 ; BERTIN et al., 1976 ; CROSs, 1977 ; GAllais et al., 1976 et 1981 ; BOYAT et al., 1983a).

Les génotypes prolifiques en épis se caractérisent par leur aptitude à former au moins un $2^{\mathrm{e}}$ épi de taille et de précocité voisines de celles de l'épi primaire à densité de culture normale ou faible. Avec une activité nitrate-réductase supérieure à celle des 
types classiques (BOYAT \& ROBIN, 1977 ; BOYAT et $a l ., 1983 \mathrm{~b}$ ), ces génotypes présentent une bonne tolérance aux forts peuplements, même si, dans ce cas, la prolificité ne s'exprime pratiquement pas (DUVICK, 1974 ; BERTIN et al., 1976 ; GUNN \& WEBB, 1977). Ils ont aussi, probablement, une teneur en protéines de la plante entière plus élevée (GaLlais et al., 1981 et 1982). En raison d'une bonne simultanéité de floraison, avec une tendance à la protogynie (BOYAT et $a l ., 1983 a)$, ces maïs assurent une fécondation convenable même en conditions sèches avec en plus, semble$\mathrm{t}$-il, une meilleure résistance intrinsèque à la sécheresse. Par ailleurs, la digestibilité des tiges de ces génotypes pourrait être supérieure à celle des types habituels (GALLAIS et al., 1981).

Enfin, et d'un point de vue méthodologique, ce type de plantes permet la réalisation des schémas de sélection récurrente réciproque plein-frères (HALLAUER, 1973), qui réalisent un bon compromis entre l'amélioration du matériel de base et la sortie en création variétale (GALLAIS et al., 1981).

Les travaux réalisés à ce jour sur du matériel prolifique ont porté essentiellement sur des types demiprécoces à tardifs. Dans le cadre des travaux d'amélioration des maïs précoces et très précoces, nous avons constitué et commencé la sélection de 2 populations prolifiques en épis, sur lesquelles différentes mesures morphologiques ont été réalisées, afin de préciser les caractéristiques particulières de ce type de génotypes.

\section{MATÉRIELS ET MÉTHODES}

\section{A. Formation des populations}

La population «à grains cornés 》 a été réalisée en croisement pyramidal entre des lignées précoces et des lignées prolifiques ou semi-prolifiques plus tardives (tabl. 1). Les hybrides multiples ont été intercroisés sans maintien cles filiations maternelles en isolement, d'abord en conditions de milieu non limitantes (Montpellier, Hérault) avec sélection massale pour la prolificité, puis en région Nord (Mons, Somme) où 287 plantes $S_{0}$ prolifiques et précoces ont été retenues (cycle de sélection $\mathrm{C}_{0}$ ).

La population "à grains dentés " a été réalisée de la même façon en croisement pyramidal entre des lignées précoces et des lignées prolifiques plus tardi- ves ou des plantes prolifiques de la synthétique prolifique tardive $\mathrm{FS}_{10}$. Les hybrides multiples ont été intercroisés avec maintien des filiations maternelles, d'abord manuellement en conditions de milieu non limitantes (St Martin-de-Hinx, Landes), puis en isolement en région Nord (Mons, Somme), avec paillage plastique, où 220 plantes $S_{0}$ prolifiques et précoces ont été retenues dans 131 familles de demi-frères ayant au moins 1,7 épi par plante en moyenne (cycle de sélection $\mathrm{C}_{0}$ ).

\section{B. Observations sur les populations}

Les lignes de chacune des familles de demi-frères issues des plantes $S_{0}$ sélectionnées ont été observées, sans répétition, à Mons respectivement en 1981 et 1982 pour les familles "cornées " et "dentées", avec une densité de culture de 80000 plantes/ha (allées de $0,80 \mathrm{~m}$ comprises, lignes de 20 plantes). Les parcelles mal levées ont été éliminées et 256 et 157 familles, respectivement cornées et dentées, ont été effectivement prises en compte. Les notations ont porté sur la date de floraison femelle, le nombre de feuilles non sénescentes à floraison situées d'une part sous l'épi supérieur, d'autre part au-dessus de l'épi supérieur (feuille de cet épi comprise), sur la surface de la feuille de l'épi supérieur (longueur $\times$ largeur au tiers inférieur $\times 0,75)$ et sur la prolificité en épis à maturité (cycle de sélection $C_{1}$ ).

\section{Estimation de l'héritabilité de la prolificité}

Une sélection a été effectuée entre les cycles $C_{0}$ et $\mathrm{C}_{1}$. Pour la population dentée conduite avec suivi des filiations maternelles, on peut toutefois estimer l'héritabilité de la prolificité, d'une part, à partir de la régression descendant/parent, d'autre part, à partir de la ressemblance entre familles de demi-frères observées lors du cycle $C_{1}$ et issues d'une même famille de demi-frères sélectionnée en $C_{0}$ (FALCONER, 1961 ; KEMPTHORNE, 1957).

Des modèles ont été développés (THOMPSON, 1973, 1977), qui permettent de prendre en compte ces 2 types d'information ainsi que l'effet de la sélection. Les composantes de la variance sont estimées par la méthode du maximum de vraisemblance selon une méthode itérative (KENDALL \& STUART, 1973).

Les hypothèses simplificatrices faites dans le cas présent sont les suivantes :

TABLEAU 1

Liste du matériel constituant les deux populations prolifiques précoces.

Inbreds used in early prolific synthetics.

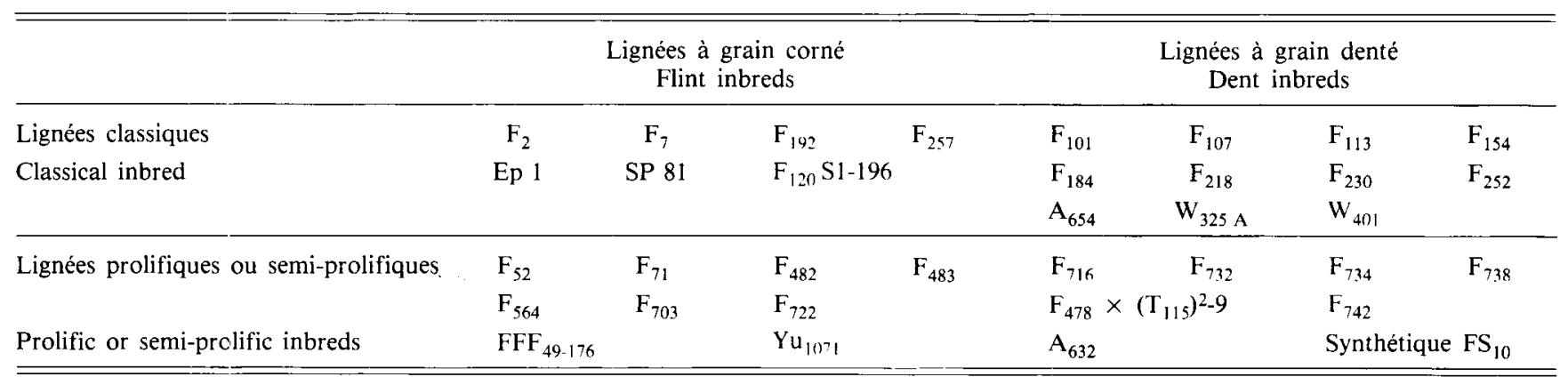


- on ne considère que les parts additives $\left(\sigma_{A}^{2}\right)$ et de dominance $\left(\sigma_{\mathrm{D}}^{2}\right)$ dans la variance génétique ;

- on suppose que la variance environnementale $\left(\sigma_{\mathrm{E}}^{2}\right)$ fut la même en $\mathrm{C}_{0}$ et en $\mathrm{C}_{1}$;

- on suppose qu'il n'y a pas d'effet milieu com$\operatorname{mun}\left(\sigma_{\mathrm{C}}^{2}=0\right)$.

Compte tenu des structures familiales impliquées, on ne peut séparer $\sigma_{\mathrm{D}}^{2}$ de $\sigma_{\mathrm{E}}^{2}$, et c'est leur somme $\sigma_{\mathrm{K}}^{2}$ qui est estimée.

L'héritabilité (au sens strict) estimée habituellement est le rapport $h^{2}=\sigma_{A}^{2} /\left(\sigma_{A}^{2}+\sigma_{K}^{2}\right)$.

On peut également s'intéresser, d'un point de vue plus pratique, au gain génétique attendu pour la méthode de sélection qui a été effectivement utilisée en prenant en compte l'apparentement des familles de demi-frères issues d'une même famille de demi-frères maternelle.

plante mère année $n$

familles de $1 / 2$ frères, année $\mathrm{n}+1$ - cycle $\mathrm{C}_{0}$

familles de $1 / 2$ frères, année $\mathrm{n}+2$ - cycle $\mathrm{C}_{1}$

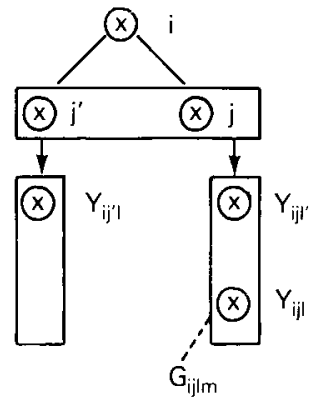

Dans ce schéma, $Y_{i j l}$ est la valeur phénotypique d'une plante observée l'année $n+2$.

On cherche à prédire (GALLAIS, 1980) $\mathrm{G}_{\mathrm{ij} \mid \mathrm{m}}$, la valeur génétique (additive) d'un de ses descendants obtenus en fécondation libre, l'ensemble des plantes participant à la fécondation en tant que mâle.

L'index de sélection $I_{i j l}$ d'une plante observée en année $\mathbf{n}+2$ s'écrit :

$$
\begin{aligned}
I_{i j l}= & \frac{\frac{1}{32} \sigma_{A}^{2}+\frac{1}{n_{i}} \frac{3}{32} \sigma_{A}^{2}+\frac{1}{N_{n}} \frac{3}{8} \sigma_{A}^{2}}{\frac{1}{16} \sigma_{A}^{2}+\frac{1}{n_{i}} \frac{3}{16} \sigma_{A}^{2}+\frac{1}{N_{n_{i}}}\left(\frac{3}{4} \sigma_{A}^{2}+\sigma_{\mathrm{K}}^{2}\right)}\left(Y_{i . .}-\mu_{2}\right)+ \\
& \frac{\frac{3}{32} \sigma_{A}^{2}+\frac{1}{N} \frac{3}{8} \sigma_{A}^{2}}{\frac{3}{16} \sigma_{A}^{2}+\frac{1}{N}\left(\frac{3}{4} \sigma_{A}^{2}+\sigma_{K}^{2}\right)}\left(Y_{i j .}-Y_{i . .}\right)+\frac{\frac{3}{8} \sigma_{A}^{2}}{\frac{3}{4} \sigma_{A}^{2}+\sigma_{K}^{2}}\left(Y_{i j l}-Y_{i j .}\right)
\end{aligned}
$$

en prenant en compte les informations relatives à cette même année ( $\mu_{2}$ étant la moyenne théorique de la population en année $n+2$ ).

Dans cette équation, $\mathrm{N}$ est le nombre moyen de plantes observées par famille de $1 / 2$ frères en année $n+2, n_{j}$ est le nombre de familles de $1 / 2$ frères issues d'une même famille ancêtre en année $n+1$.

Le gain génétique $\Delta \mathrm{G}$ obtenu en un cycle de sélection s'écrit (RouvIER, 1969): $\Delta \mathrm{G}=\mathrm{i} . \sigma_{1}$ où $\mathrm{i}$ est l'intensité de sélection et $\sigma_{\mathrm{I}}$ est l'écart-type de l'index $I_{i j l}$.

\section{RÉSULTATS - DISCUSSION}

Les caractéristiques des 2 populations sont rassemblées dans le tableau 2. La plus faible variabilité intra-population du matériel "denté » pour la date de floraison est sans doute aussi une conséquence des conditions climatiques plus chaudes de l'année 1982. Le niveau de prolificité moyen de ces familles de demi-frères est le même pour les 2 populations avec cependant une variabilité plus importante pour la population " cornée ». Les nombres de feuilles sous l'épi supérieur sont pratiquement les mêmes dans les 2 cas, le nombre un peu plus faible observé pour la. population « dentée » étant probablement dû, en partie au moins, à une sénescence plus rapide des feuilles de la base des plantes en 1982 . En revanche, les plantes de la population «dentée » présentent presque 1,5 feuille de plus au-dessus de l'épi que les plantes de la population cornée, résultat parallèle à celui de PHILIPPE (1980), sans que cette différence puisse ici être expliquée essentiellement par une différence de précocité des 2 populations. De même, la surface de la feuille de l'épi supérieur est presque $1,5 \mathrm{dm}^{2}$ plus grande pour les plantes à grain denté. Pour ces 3 derniers caractères, les écarts-type sont les mêmes pour les 2 populations.

L'étude des corrélations et des régressions des différents caractères observés sur la prolificité permet de préciser les caractéristiques du matériel prolifique

TABLEAU 2

Caractéristiques moyennes des descendances demi-frères des plantes $S_{0}$ choisies dans les populations prolifiques précoces.

\begin{tabular}{|c|c|c|c|c|c|c|}
\hline \multirow[b]{3}{*}{ Familles à grain corné } & \multirow[b]{3}{*}{ moyenne } & \multirow{3}{*}{$\begin{array}{c}\text { Prolificité } \\
\text { Prolificacy } \\
1,42\end{array}$} & \multirow{3}{*}{$\begin{array}{c}\begin{array}{c}\text { Date de floraison } \\
\text { femelle } \\
\text { Silking date } \\
\text { (août-august) }\end{array} \\
7,84\end{array}$} & \multirow{2}{*}{\multicolumn{2}{|c|}{$\begin{array}{l}\text { Nombre de feuilles } \\
\text { Number of leaves } \\
\text { sous au-dessus de } \\
\text { below above } \\
\text { l'épi supérieur } \\
\text { the first ear }\end{array}$}} & \multirow{3}{*}{$\begin{array}{c}\begin{array}{c}\text { Surface feuille } \\
\text { épi supérieur } \\
\text { First ear leaf } \\
\text { area }\end{array} \\
5,00\end{array}$} \\
\hline & & & & & & \\
\hline & & & & 5,87 & 5,37 & \\
\hline $\begin{array}{l}\text { Flint families } \\
\text { (Mons-1981) }\end{array}$ & $\begin{array}{l}\text { écart-type } \\
256 \text { familles }\end{array}$ & 0,63 & 2,65 & 0,62 & 0,35 & 0,47 \\
\hline Famille à grain denté & moyenne & 1,40 & 0,96 & 5,53 & 6,63 & 6,38 \\
\hline $\begin{array}{l}\text { Dent families } \\
\text { (Mons-1982) }\end{array}$ & $\begin{array}{l}\text { écart-type } \\
157 \text { familles }\end{array}$ & 0,29 & 0,21 & 0,59 & 0,33 & 0,48 \\
\hline
\end{tabular}
Average values for half-sib progenies of $S_{0}$ selected plants in early prolific synthetics. 
(fig. 1). La liaison prolificité $\times$ tardiveté existe seulement dans la population cornée, et ceci est sans doute à relier à la plus grande variabilité de cette population à la fois pour la précocité et la prolificité. De même, la surface de la feuille de l'épi supérieur tend à être plus élevée dans les familles prolifiques seulement pour le matériel corné, en raison également, et pour une partie au moins, de cette liaison tardiveté $\times$ prolificité. Le nombre de feuilles situées audessus de l'épi supérieur est pratiquement le même pour les plantes prolifiques et non prolifiques dans les 2 populations, avec toutefois une tendance à un nombre légèrement plus faible $(-0,25$ feuille par plante) pour les plantes prolifiques de la population dentée. En revanche, les plantes prolifiques possèdent pratiquement une feuille supplémentaire située au-

A

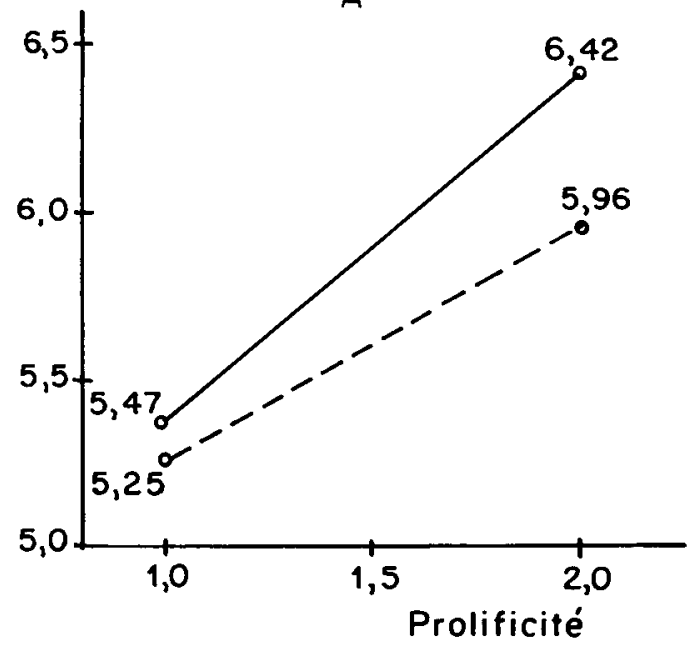

Nombre de feuilles sous l'épi supérieur Number of leaves below the first ear corné - flint $r=0,30 \quad r p=N S$ denté - dent $r=0,35 \quad r p=0,36$

C

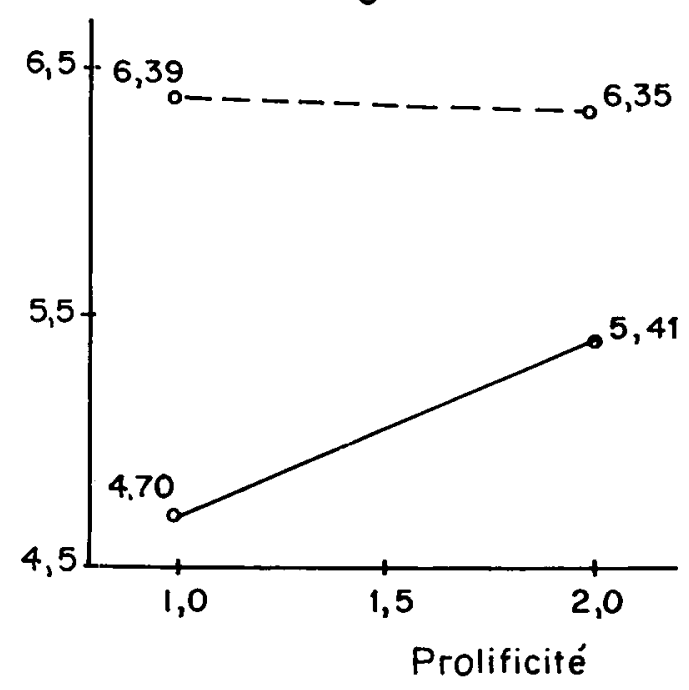

Surface de la feuille de l'épi supérieur $\left(\mathrm{dm}^{2}\right)$ First ear leaf area $\left(\mathrm{dm}^{2}\right)$

$$
\begin{array}{lll}
\text { corné - flint } r=0,30 & r p=N S \\
\text { denté - dent } r=N S & r p=N S
\end{array}
$$

Figure 1

Observations des familles issues des $S_{0}$ des populations prolifiques précoces.

Notations of half-sib families from early prolific synthetics. ○— Mons, 198 ! ; familles à grain corné - flint families ○--- -0 Mons, 198?; familles à grain denté - dent families dessous de l'épi supérieur par rapport aux plantes non prolifiques (respectivement 0,95 et 0,71 feuille supplémentaire pour les populations «cornées » et " dentées »), caractère encore lié à la précocité pour le matériel "corné ». PHILIPPE (1980) avait obtenu des résultats semblables sur du matériel un peu plus tardif (respectivement 0,9 et 0,7 feuille supplémentaire chez les plantes prolifiques des populations $\mathrm{FS}_{11}$ (" cornée ») et $\mathrm{FS}_{10}$ (« dentée »). SoRRELS et al. (1979) observaient également une corrélation positive significative entre la prolificité et le nombre de nœuds situés sous l'épi supérieur ainsi qu'une corrélation négative significative entre la prolificité et le nombre de nœuds situés au-dessus de l'épi supérieur, observations réalisées sur du matériel « denté » à base génétique étroite, et à faible densité. Enfin, PRIOR \& RUS-

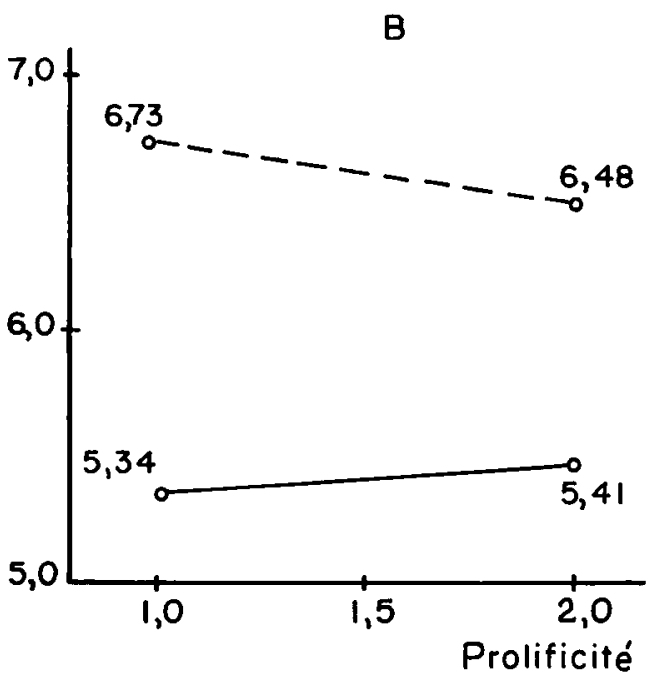

Nombre de feuilles au-dessus de l'épi supérieur Number of leaves above the first ear

$$
\text { corné - flint } r=N S \quad r p=N S
$$$$
\text { denté - dent } r=-0,23 \mathrm{rp}=-0,22
$$

$\mathrm{D}$

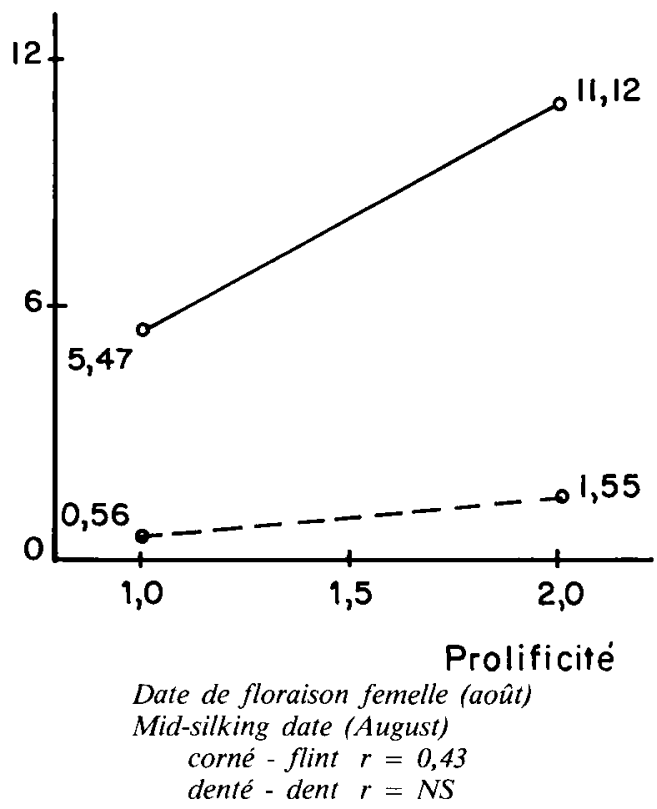

$r=$ corrélation entre la prolificité et le caractère observé i $S$ à I p. 100) correlation between prolificacy and observed character $(1 \%$ level)

$r p=$ corrélation partielle à précocité fixée partial correlation at fixed earliness. 
SEL (1975) observaient des indices foliaires (LAI) supérieurs chez des hybrides prolifiques comparés à des types classiques.

Pour la population "dentée ", et en utilisant la méthode présentée précédemment, les estimations de $\sigma_{\mathrm{A}}^{2}$ et $\sigma_{\mathrm{K}}^{2}$ sont :

$$
\begin{aligned}
& \sigma_{\mathrm{A}}^{2}=0,180 \pm 0,010 \\
& \sigma_{\mathrm{K}}^{2}=0,533 \pm 0,074,
\end{aligned}
$$

ce qui donne une héritabilité au sens strict $\mathrm{h}^{2}=0,25$. Dans le dispositif installé en 1982, la ressemblance entre demi-frères peut être surestimée puisque les individus d'une même famille étaient cultivés sur une seule et même ligne (variance de l'effet milieu commun). La méthode d'estimation utilisée, qui prend en compte la relation parent-descendant et met en jeu des mesures effectuées au cours de 2 années différentes, tend vraisemblablement à diminuer ce biais. L'héritabilité de la prolificité observée ici est la même que celle obtenue en moyenne par HALLAUER (1974) à partir d'hybrides simples tardifs, à différentes densités, tous croisements et densités confondus $\left(\mathrm{h}^{2}=0,24\right)$. Il soulignait toutefois la très forte variation des valeurs d'héritabilité observée entre hybrides ou entre densités.

Avec 2 descendances retenues $\left(n_{i}=2\right)$ dans des familles sélectionnées de 20 plantes $(\mathrm{N}=20)$, effectifs qui correspondent grossièrement aux conditions utilisées lors du tri, l'index $\mathrm{I}_{\mathrm{ijl}}$ s'écrit :

$$
\begin{gathered}
I_{i j l}=0,351\left(Y_{i .}-\mu_{2}\right)+0,301\left(Y_{i j}-Y_{i . .}\right)+ \\
+0,10\left(Y_{i j l}-Y_{i j}\right) .
\end{gathered}
$$

Les coefficients de l'index montrent l'importance du choix des meilleures familles par rapport au choix des meilleures plantes dans les familles, sachant par ailleurs que, jusqu'alors, seules des plantes bien prolifiques ont été récoltées $\left(Y_{i j l}=2\right.$, exceptionnellement 3).

La figure 2, qui donne l'évolution attendue du gain génétique en fonction du dispositif décrit par le couple $\left(n_{i}, N\right)$ montre que, pour $n_{i}=2$ et $N=20$, on peut attendre un gain voisin de 0,12 épi par plante par cycle de sélection, en prenant $i=1$, ce qui correspond à un taux de sélection de $1 / 3$. Elle confirme aussi qu'un effectif de 20 plantes par famille est suffisant, mais montre l'intérêt de retenir au moins 2 descendances dans les familles sélectionnées (ce qui est tout à fait réalisable en pratique avec la même intensité de sélection), alors que nous n'en avons retenu que 1,53 en moyenne. Par ailleurs, ce gain génétique attendu semble supérieur à ceux obtenus, sur du matériel différent et dans d'autres conditions de culture, par MIES (1979) ou par DE MIRANDA et al. (1978). Ceci est peut-être dû à nos conditions de sélection à forte densité de culture.

Il est également possible de prendre en compte dans le calcul d'index la valeur des familles-mères correspondantes. L'index obtenu s'écrit alors

$$
\begin{aligned}
& \mathrm{I}_{\mathrm{ijl}}=0,055\left(\mathrm{x}_{\mathrm{i} .}-\mu_{1}\right)+0,317\left(\mathrm{Y}_{\mathrm{i} . .}-\mu_{2}\right)+ \\
& \quad+0,301\left(\mathrm{Y}_{\mathrm{ij} .}-\mathrm{Y}_{\mathrm{i} .}\right)+0,10\left(\mathrm{Y}_{\mathrm{ijl}}-\mathrm{Y}_{\mathrm{ij} .}\right)
\end{aligned}
$$

$\mu_{1}$ étant la moyenne des familles-mère, les 2 derniers coefficients étant inchangés. Ce calcul montre en fait que les choix faits avec les 2 index sont les mêmes,

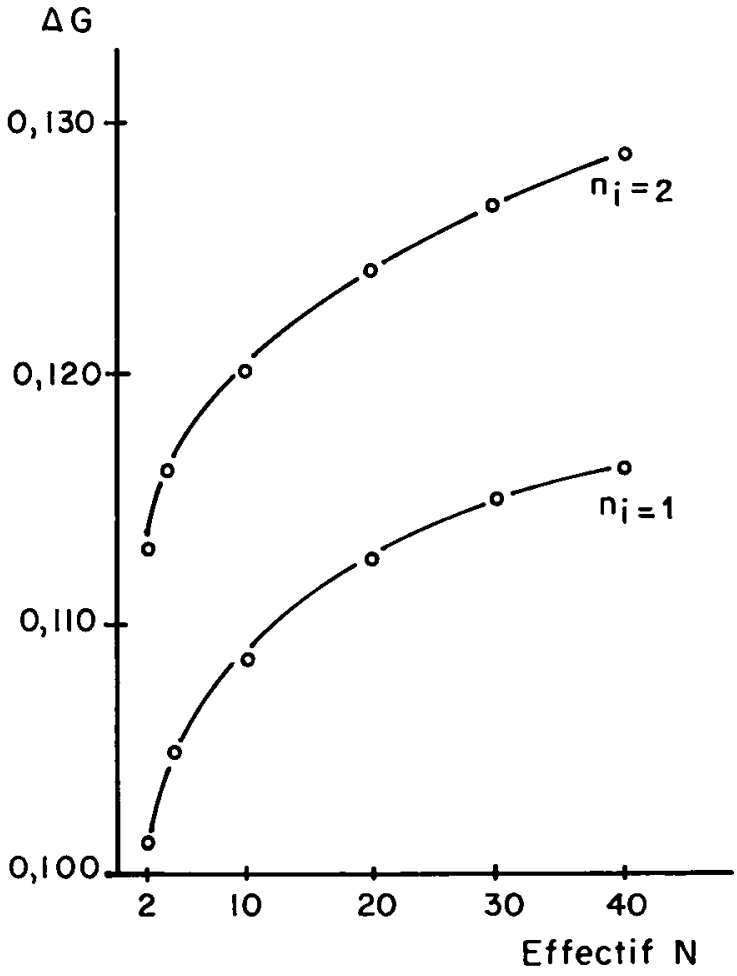

Figure 2

Gain génétique attendu par cycle de sélection pour la prolificité en fonction des effectifs $\left(N, n_{i}\right)$ pour une intensité de sélection $i=I$. Effectif $N$ : nombre de plantes par famille.

$\Delta G:$ gain génétique par cycle pour une intensité de sélection $i=l$.

Expected genetic progress per selection cycle for ear prolificacy with varying value of $\left(N, n_{i}\right)$; selection intensity $i=1$. Effectif $N$ : number of plants per family

$\Delta G:$ genetic gain per cycle with a selection rate $i=1$.

l'information apportée par les familles-mère étant affectée d'un très faible coefficient.

La corrélation entre la prolificité des familles-mère et la moyenne des descendances de ces familles est significative, mais relativement faible $r=0,31$. Toutefois, cela n'est pas contradictoire avec l'importance du choix familial, puisque cette corrélation est calculée dans un intervalle plus étroit de variation pour la prolificité (familles sélectionnées). Par ailleurs, même si le déterminisme génétique de la prolificité n'est pas parfaitement compris, il semble bien établi que la prolificité est un caractère polygénique à seuil (HALLAUER, 1974), à tendance récessive (BONCIARELLI, 1964 ; BERTIN et al., 1976 ; HARRIS et al., 1976), déterminisme qui contribue à expliquer l'héritabilité relativement faible $\mathrm{du}$ caractère dans les familles sélectionnées.

\section{CONCLUSION}

Cette étude, qui nous a permis de choisir pour le cycle suivant 53 familles prolifiques dans chacune des populations « cornées » et «dentées », montre qu'il est possible de sélectionner du matériel précoce, prolifique en épis à forte densité de culture. Les calculs d'index confirment l'intérêt d'une sélection combinée individuelle et familiale, conduisant à un gain génétique relativement élevé par cycle de sélection pour la prolificité. La présence d'un nœud supplémentaire est 
confirmée chez les plantes prolifiques, dont l'une des conséquences est d'augmenter la hauteur des plantes. Cette plus grande taille, avec des épis insérés haut, peut expliquer en partie au moins, la sensibilité assez élevée de ces familles à la verse en végétation, défaut d'ailleurs caractéristique de certains des géniteurs de prolificité utilisés. L'amélioration de ces populations est envisagée selon un modèle récurrent réciproque parallèle à celui proposé par GALLAIS (1977), qui comprend un axe central d'amélioration des populations avec une intensité de sélection modérée et la préservation de la variabilité, des sorties en création variétale par des sous-populations à forte intensité de sélection et un axe parallèle de populations tampon qui permet l'introduction et l'adaptation de variabilité nouvelle. L'amélioration du niveau de résistance à la verse et l'introduction de nouvelles sources de prolificité se feront donc par l'intermédiaire de ces populations tampons, sans régression trop importante des performances des populations centrales.

Reçu le 27 juin 1983. Accepté le 5 décembre 1983.

\section{RÉFÉRENCES BIBLIOGRAPHIQUES}

Bertin C., Panouillé A., Rautou S., 1976. Obtention de variétés de maïs prolifiques en épis, productives en grains, à large adaptation écologique. Ann. Amélior. Plantes, 26 (3), 387-418.

Bonciarelli F., 1964. Osservazioni sperimentali sulla prolificita del maïs (Zea mays L.). Quaderno 3 di Maydica, Anno 9, Publicazione 170 .

Boyat A., Robin P'., 1977. Relations entre productivité, qualité du grain et activité nitrate réductase chez les céréales. Ann. Amélior. Plantes, 27 (4), 389-410.

Boyat A., Ramdoyal K., Robin P., Kaan F., 1983a. Prolificité en épi et activité nitrate réductase. Colloque Physiologie du mais, Royan, 16 mars 1983.

Boyat A., Ramdoyal K., Kaan F., Panouillé A., 1983b. Densité de peuplement et prolificité en épis. Colloque Physiologie du mais, Royan, 15 mars 1983.

Cross H. Z., 1977. Interelationships among yield stability and yield components in eary maize. Crop Sci., 17, 741-745.

De Miranda L. Torres, De Miranda L. E. Coelho, Pommer C. V., Sawazaki E., 1978. Melhoramento genético do cultivar de milho IAC-1. Bragantia, 37 (8), 63-70.

Duvick D. N., 1974. Continuous back-crossing to transfer prolificacy to a single eared inbred line of maize. Crop Sci., 14, 69-71.

El-Lakany M. A., Russel W. A., 1971. Relationship of maize characters with yield in test-crosses of inbreds at different plant densities. Crop Sci., 11, 698-701.

Falconer D. S., 1961. Introduction to quantitative genetics. Oliver and Boyd, $365 \mathrm{p}$.

Gallais A., 1977. Stratégie d'amélioration des populations à large base génétique. Ann. Amélior. Plantes, 27 (3), 281-329.

Gallais A., 1980. Sélection combinée et plans de croisements en amélioration des plantes. I. Eléments théoriques pour quelques plans équilibrés. Ann. Amélior. Plantes, 30 (4), 411-441.

Gallais A., Pollacsek M., Huguet L., 1976. Possibilités de sélection du maïs en tant que plante fourragère. Ann. Amélior. Plantes, 26 (4), 591-605.

Gallais A., Vincourt P., Huguet L., 1981. Objectifs et critères de sélection du maïs fourrage. Congrès Eucarpia, Montreux, septembre 1981.

Gallais A., Kellerhals M., Philippe F., 1982. Etude des interactions entre épis chez le rnaîs. Agronomie, 2 (10), 995-1004.

Gunn R. W., Webb P. J., 1977. Maize. Ann.-Rep. Plant Breed. Inst. Cambridge, Ann. Rep., 1977.
Hallauer A. R., 1973. Hybrid development and population improvement in maize by reciprocal full-sib selection. Egypt. J. Genet Cytol., 2, 84-101.

Hallauer A. R., 1974. Heritability of prolificacy in maize. The $J$ of Hered., 65, 163-168.

Hallauer A. R., Troyer A. F., 1972. Prolific corn hybrids and minimizing risk of stress. Annu-Hybrid Corn Industry Res. Conf Proc., 27, 140-157.

Harris R. E., Moll R. M., Stuber C. W., 1976. Control and inheri tance of prolificacy in maize. Crop Sci., 16, 843-850.

Kempthorne O., 1957. An introduction to genetic statistics. John Wiley and Sons, 545 p.

Kendall M. G., Stuart A., 1973. The advanced theory of statistics Charles Griffin, $3^{\text {e }}$ édition, volume 2

Mies D. W., 1979. Effects of mass selection for early maturity and prolificacy on epistasis in Golden Glow maize. Diss. Abstr. Int., B 39 (8), $3631 \mathrm{~B}$.

Philippe F., 1980. Contribution à l'étude de la prolificité en épis chez le mais. Mémoire DAA, EnSA Montpellier, I.N.R.A. Lusignan $83 \mathrm{p}$.

Prior C. L., Russell W. A., 1975. Yield performance of nonprolific and prolific maize hybrids at six plant densities. Crop Sci., 15, $482-486$

Rouvier R., 1969. Contribution à l'étude des index de sélection sur plusieurs caractères. Thèse de $3^{\mathrm{e}}$ cycle, Fac. Sci., Paris, $100 \mathrm{p}$.

Russell W. A., 1968. Test crosses of one and two-ear types of corn belt maize inbreds. I. Performance at four plant stand densities. Crop Sci., 8, 244-247.

Russell W. A., Eberhart S. A., 1968. Test crosses of one and two ear types of corn belt maize inbreds. II. Stability of performance in different environments. Crop Sci., 8, 248-251.

Sorrells M. E., Lonnquist J. M., Harris R. E., 1979. Inheritance of prolificacy in maize. Crop Sci., 19, 301-306.

Thompson R., 1973. The estimation of variance and covariance components with an application when records are subjects to culling. Biometrics, 29, 527-550.

Thompson R., 1977. The estimation of heritability with unbalanced data. I. Observations available on parents and offspring. Biometrics, 33, 485-495. 